\title{
EFEITO DE IDADE E SÍTIO NO AJUSTE DE FUNÇÕES PROBABILÍSTICAS PARA ALTURA DE Mimosa scabrella
}

\author{
Ronan Felipe de Souza ${ }^{1}$, Rodrigo Geroni Mendes Nascimento ${ }^{1}$, Sylvio Péllico Netto ${ }^{2}$, \\ Sebastião do Amaral Machado ${ }^{2}$
}

\begin{abstract}
${ }^{1}$ Eng. Florestal, Doutorando em Eng. Florestal, UFPR, Curitiba, PR, Brasil - ronanflorestal@ gmail.com; geronimendes@ hotmail.com ${ }^{2}$ Eng. Florestal, Dr., Depto. de Ciências Florestais, UFPR, Curitiba, PR, Brasil - sylviopelliconetto@gmail.com; samachado@ufpr.br

Recebido para publicação: 12/12/2011 - Aceito para publicação: 06/05/2013
\end{abstract}

\begin{abstract}
Resumo
Este trabalho teve como objetivo avaliar o efeito do sítio e da idade na distribuição de alturas de bracatingais nativos da Região Metropolitana de Curitiba, bem como selecionar uma Função Densidade de Probabilidade (FDP), dentre seis, que melhor represente o histograma da variável altura desses povoamentos. Foram utilizados dados de altura provenientes de 88 parcelas de área variável de 100 a $400 \mathrm{~m}^{2}$, em três classes de sítio, nas idades de 5, 8, 9 e 13 anos, nos quais se efetuaram o ajuste das funções probabilísticas. O ajuste dos modelos foi realizado com auxílio do aplicativo Solver do Microsoft Excel 2007, pelo método dos mínimos quadrados. As FDPs testadas foram Beta, Gamma, Log Normal, Normal, Sb de Jonhson e Weibull, sendo selecionada a de melhor ajuste pelo teste de Kolmogorov-Smirnov, Índice de Schlaegel e Erro Padrão da Estimativa Percentual. O sítio e a idade afetam a amplitude, a forma e a dinâmica da distribuição de alturas, sendo o sítio a variável relacionada à curtose e a idade à assimetria da distribuição. A FDP que melhor descreveu a distribuição de alturas dos bracatingais foi a função Beta, seguida pela Sb de Johnson e Normal.

Palavras-chave: Bracatinga; distribuição hipsométrica; mínimos quadrados.
\end{abstract}

\begin{abstract}
Effect of age and site on the height distribution of Mimosa scabrella. This study aimed to evaluate the effect of site and age on the height distribution in native stands of "bracatinga" (Mimosa scabrella Benth) on the Metropolitan Region of Curitiba, PR, Brazil, as well as to select a Probability Density Function (pdf), between six, that represents the histogram of the variable height of these stands. It was used height data from 88 plots with variable area from 100 to $400 \mathrm{~m}^{2}$, classified into three site classes. The ages 5, 8, 9 and 13 years were selected for fitting probability density functions by each site. The adjustment of the models was performed using the Solver application of Microsoft Excel 2007, by the least squares method. The pdfs tested were Beta, Gamma, Log Normal, Normal, Weibull and Sb Johnson, being selected the best by Kolmogorov-Smirnov test, Schlaegel Index and Percentage Standard Error of Estimate. The site and age affect the amplitude, shape and dynamics of the height distribution, with the site related to the kutosis and age to skewness of the distribution. The pdf that best described height distribution of the native stands of "bracatinga" was Beta function, followed by Sb Johnson and Normal.

Keywords: Bracatinga; hypsometric distribution; least squares.
\end{abstract}

\section{INTRODUÇÃO}

A Mimosa scabrella Benth. (bracatinga) é uma espécie de grande aptidão para a colonização de áreas abertas e bem iluminadas, sendo heliófila em todas as fases de desenvolvimento. No interior da floresta, regenera-se somente em grandes áreas de distúrbio, como clareiras ou estradas (CARPANEZZI et al., 1988). Sua área de ocorrência natural compreende os estados do Paraná e Santa Catarina, onde é mais abundante, e com menos frequência nos estados do Rio Grande do Sul, São Paulo, Minas Gerais e Rio de Janeiro (TÉO, 2009).

O manejo tradicional da espécie é dependente da queima de resíduos de exploração florestal do ciclo anterior, sendo que seu manejo pode ser conduzido de duas formas: florestas homogêneas puras, altamente adensadas, e sistemas agroflorestais com culturas de ciclo curto nos primeiros anos, principalmente milho e feijão. Essas formas de manejo são mantidas nesses moldes na maioria das 
propriedades rurais da Região Metropolitana de Curitiba (RMC), como pode ser constatado em Baggio et al. (1986); Baggio (1994), Steenbock (2009), Gonçalves (2011) e Silva (2013), que estudaram essas práticas de manejo e as consideraram atuais. Devido a essas diferentes formas de condução dos bracatingais, os plantios da espécie, mesmo sendo equiâneos, apresentam heterogeneidade estrutural no que se refere à distribuição horizontal e vertical dos indivíduos.

O manejo de qualquer espécie ou floresta passa necessariamente pelo estudo da distribuição de frequências das variáveis dendrométricas, sendo essa análise de grande utilidade na avaliação da estrutura horizontal e vertical de povoamentos florestais. Esse ferramental é importante na análise do crescimento e desenvolvimento florestal; no diagnóstico de flutuações populacionais causadas por doenças ou pragas, mortalidade, exploração no passado; no entendimento do estágio de sucessão; na estratificação da floresta para diferentes formas possíveis de manejo; e em estudos fitossociológicos e de melhoramento genético.

Segundo Puig (2008), o uso de modelos e representações dessa organização permite analisar os subprocessos na aparente desordem de eventos que condicionam a distribuição das espécies. O estudo da distribuição da altura total de indivíduos arbóreos ainda é direcionado a trabalhos científicos, e sua utilização na avaliação de ativos florestais, para fins de manejo, é incipiente ou inexistente, devido ao custo de obtenção dessa variável em larga escala. Vários trabalhos estudaram a distribuição da variável altura na avaliação da estrutura vertical de povoamentos e florestas (FELFILI, 1997; ALVES JÚNIOR et al., 2007; PALUDO et al., 2009; GOMIDE et al., 2009), bem como no uso de funções probabilísticas para a descrição da distribuição dessa variável (WEBER, 2006; MACHADO et al., 2010; KOEHLER et al., 2010). Entretanto, não há uma bibliografia que apresente os efeitos da qualidade do sítio e da idade na distribuição hipsométrica e no ajuste de funções que a descrevam.

Machado et al. (2001) estudaram o efeito da densidade inicial e sítio em bracatingais da RMC, identificando uma influência significativa dessas variáveis sobre o diâmetro médio e, como esperado, pouca influência da densidade na altura média do povoamento. Bartoszeck et al. (2004), visando um aprofundamento desse estudo, pesquisaram o efeito da idade, sítio e da densidade na distribuição diamétrica de bracatingais, destacando que todas as variáveis afetaram significativamente a forma, amplitude, bem como o modelo a ser usado para descrição da distribuição diamétrica.

O efeito significativo do sítio sobre a altura foi apresentado por Machado et al. (2001), porém a intensidade desse efeito sobre todos os indivíduos do povoamento pouco se sabe. Machado et al. (2001) e Bartoszeck et al. (2004) avaliaram o efeito da idade e sítio na estrutura horizontal dos bracatingais, desconsiderando a amplitude da distribuição vertical dos indivíduos na análise, sendo essa última uma característica diagnóstica de povoamentos, assim como a distribuição diamétrica.

Nesse contexto, o objetivo deste trabalho foi avaliar o efeito do sítio e idade na distribuição de alturas de bracatingais nativos da RMC, identificando o efeito dessas variáveis na forma e amplitude, bem como na escolha da função densidade de probabilidade que melhor represente os histogramas da altura nas diferentes idades.

\section{MATERIAL E MÉTODOS}

Os dados utilizados provieram de 88 parcelas temporárias amostradas em povoamentos equiâneos, manejados na forma tradicional da RMC, com área variando de 100 a $400 \mathrm{~m}^{2}$, distribuídas nas idades de 5, 8, 9 e 13 anos, em três classes de sítio. A classificação de sítios dos povoamentos amostrados foi realizada de acordo com Machado et al. (2011) (Tabela 1). As idades foram determinadas por contagem dos anéis de crescimento, realizada por meio da coleta de disco a $10 \mathrm{~cm}$ da base da árvore ou pela retirada de rolo de incremento com trado de Pressler.

No total, foram amostradas 3.971 valores de alturas, mensuradas com uma vara graduada de $12 \mathrm{~m}$ ou com um Blume-Leiss. A base de dados foi agrupada em 8 classes de altura e amplitude de 3 metros; os centros de classe variaram de $2,50 \mathrm{~m}$ a $23,5 \mathrm{~m}$. O agrupamento dos dados foi realizado de forma empírica, atendendo à premissa de que as classes de altura devem comportar todos os dados, independentemente da idade ou classe de sítio analisada. $\mathrm{O}$ ajuste de funções de densidade de probabilidade (FDP) foi realizado para cada sítio nas 4 idades avaliadas, permitindo, assim, a obtenção da distribuição das alturas nos diferentes níveis de produtividade ao longo do ciclo de corte utilizado no manejo tradicional da espécie.

As seis funções de densidade de probabilidade testadas com respectivas restrições são apresentadas na tabela 2. Utilizando os dados não agrupados, o ajuste das funções foi realizado com auxílio do aplicativo do software Microsoft Excel 2007, denominado Solver. O ajuste das funções foi realizado pelo método dos mínimos quadrados. 
Tabela 1. Número de parcelas e indivíduos por classe de idade e classe de sítio utilizadas na modelagem da distribuição de alturas de bracatingais nativos da Região Metropolitana de Curitiba, PR, Brasil.

Table 1. Number of plots and trees per age class and site class used in modeling of the altimetric distribution in native stands of "bracatinga" (Mimosa scabrella) on the Metropolitan Region of Curitiba, PR, Brazil.

\begin{tabular}{|c|c|c|c|c|c|c|c|c|}
\hline \multirow{3}{*}{$\begin{array}{l}\text { Idade } \\
\text { (anos) }\end{array}$} & \multicolumn{6}{|c|}{ Classes de sítio } & \multirow{2}{*}{\multicolumn{2}{|c|}{ Total }} \\
\hline & \multicolumn{2}{|r|}{ I } & \multicolumn{2}{|c|}{ II } & \multicolumn{2}{|c|}{ III } & & \\
\hline & Parcelas & Indivíduos & Parcelas & Indivíduos & Parcelas & Indivíduos & Parcelas & Indivíduos \\
\hline 5 & 8 & 479 & 14 & 1.273 & 1 & 45 & 23 & 1.797 \\
\hline 8 & 6 & 181 & 10 & 478 & 8 & 436 & 24 & 1.095 \\
\hline 9 & 10 & 288 & 9 & 319 & 4 & 153 & 23 & 760 \\
\hline 13 & 5 & 74 & 11 & 197 & 2 & 48 & 18 & 319 \\
\hline Total & 29 & 1.022 & 44 & 2.267 & 15 & 682 & 88 & 3.971 \\
\hline
\end{tabular}

Após agrupamento dos dados nas 8 classes adotadas, a seleção da melhor função de densidade de probabilidade foi realizada pelo teste de Kolmogorov-Smirnov, análise das estatísticas de ajuste e precisão do Índice de Schlaegel (1981), denominado IA, também conhecido como $\mathrm{R}^{2}$ ajustado e corrigido, e o Erro Padrão da Estimativa Percentual $\left(\mathrm{S}_{\mathrm{yx}} \%\right)$.

Tabela 2. Funções densidade de probabilidade testadas e respectivas restrições.

Table 2. Tested probabilistic density functions and respective restrictions.

\begin{tabular}{|c|c|c|}
\hline Nome & Função densidade de probabilidade & Restricões \\
\hline Beta & $f(x)=\frac{\Gamma(\alpha+\beta)}{\Gamma(\alpha) \Gamma(\beta)} \cdot \frac{1}{\left(x_{\max }-x_{\min }\right)^{\alpha+\beta-1}} \cdot\left(x-x_{\min }\right)^{\alpha-1} \cdot\left(x_{\max }-x\right)^{\beta-1}$ & $\begin{array}{c}x_{\min }<x<x_{\max } \\
\quad \alpha, \beta>0 \\
-\infty<x_{\min }<x_{\max }<+\infty\end{array}$ \\
\hline Gamma & $f(x)=\frac{\left(x-x_{\min }\right)^{\alpha-1} \cdot e^{\frac{-\left(x-x_{\min }\right)}{\beta}}}{\beta^{\alpha} \cdot \Gamma(\alpha)}$ & $\begin{array}{c}x \geq x_{\min } \\
\alpha, \beta>0 \\
-\infty<x_{\min }<+\infty\end{array}$ \\
\hline $\begin{array}{l}\text { Log } \\
\text { Normal }\end{array}$ & $f(x)=\frac{e^{-\frac{1}{2}\left(\frac{(L n(x)-\mu)}{\sigma}\right)^{2}}}{\sqrt{2 \pi \sigma^{2}}}$ & $\begin{aligned} x & \geq x_{\min } \\
& \sigma>0 \\
-\infty & <x<+\infty \\
-\infty & <\mu<+\infty\end{aligned}$ \\
\hline Normal & $f(x)=\frac{1}{\sigma \sqrt{2 \pi}} e^{-\frac{1}{2}\left(\frac{x-\mu}{\sigma}\right)^{2}}$ & $\begin{aligned} \sigma & >0 \\
-\infty & <x<+\infty \\
-\infty & <\mu<+\infty\end{aligned}$ \\
\hline $\begin{array}{l}\text { Sb de } \\
\text { Johnson }\end{array}$ & $f(x)=\frac{\delta}{\sqrt{2 \pi}} \cdot \frac{\lambda}{(x-\varepsilon)(\lambda+\varepsilon-x)} e^{-\frac{1}{2}\left[\left(\gamma+\delta \ln \frac{x-\varepsilon}{\lambda+\varepsilon-x}\right)\right]^{2}}$ & $\begin{array}{c}\mathcal{E}<x<+\lambda \\
-\infty<\gamma<+\infty \\
\lambda, \delta>0\end{array}$ \\
\hline Weibull & $f(x)=\frac{\delta}{\beta}\left(\frac{x-\alpha}{\beta}\right)^{\delta-1} e^{-\left(\frac{x-\alpha}{\beta}\right)^{\delta}}$ & $\begin{array}{l}x \geq x_{\min } \\
-\infty<x_{\min }<+\infty \\
\beta, \delta>0 \\
\alpha \leq x^{2}\end{array}$ \\
\hline
\end{tabular}

$\mathrm{f}(\mathrm{x})$ : função densidade da variável $\mathrm{x}$; $\mathrm{x}$ : variável aleatória; $\mathrm{x}_{\max }=$ valor máximo de $\mathrm{x} ; \mathrm{x}_{\min }=$ valor mínimo de $\mathrm{x} ; \mu=$ média paramétrica de $\mathrm{x} ; \sigma=$ desvio padrão de $\mathrm{x} ; \pi$ : constante pi $(3,1416 \ldots) ; \alpha, \beta, \mathcal{E}, \lambda, \delta, \gamma=$ coeficientes a serem estimados.

\section{RESULTADOS E DISCUSSÃO}

Os resultados obtidos no teste Kolmogorov-Smirnov são apresentados na tabela 3. Dentre os 72 ajustes realizados, metade foi aderente quando submetidos ao teste de Kolmogorov-Smirnov. No geral, o modelo de distribuição Beta foi o que apresentou ajustes de melhor aderência aos dados, seguido por $\mathrm{Sb}$ de 
Johnson e modelo de distribuição Normal. O modelo Log Normal apresentou o pior desempenho, sendo o menos indicado na modelagem da distribuição de alturas para os povoamentos de bracatinga avaliados.

No quinto ano, para a distribuição de alturas do sítio I, bem como para o oitavo ano no sítio II, nenhum modelo foi aderente segundo o teste de Kolmogorov-Smirnov, entretanto os três modelos mais precisos na descrição do histograma de frequência para esses anos foram, respectivamente Weibull, Beta e Normal, apresentando para ambos os sítios e estratos valores de IA acima de 0,99 e erros abaixo de 5\%. Os resultados particulares a esses sítios e anos não se repetem nos demais cenários para a função de Weibull, que perde em desempenho nessas estatísticas de ajuste e precisão, dando lugar ao modelo $\mathrm{Sb}$ de Johnson, que assume bons desempenhos em todos os anos e classes de sítio (Tabela 3 e 4).

Machado et al. (2010) testaram a eficiência de funções probabilísticas para estimar a frequência de indivíduos de Araucaria angustifolia Benth. O. Kuntze. em classes de altura definidas a partir de 3 métodos exploratórios de dados. O resultado encontrado indicou que as funções Normal, Weibull e Sb de Johnson apresentaram os melhores ajustes para a distribuição da altura da espécie, porém alternando de posição de acordo com o número de classes adotado.

Tabela 3. Valores do teste de Kolmogorov-Smirnov de todos os modelos ajustados por idade e sítio. Table 3. Values of Kolmogorov-Smirnov test for each adjusted model and by age and site.

\begin{tabular}{llcccc}
\hline \multirow{2}{*}{ Sítio } & Função probabilística & \multicolumn{3}{c}{ Anos } \\
\cline { 3 - 6 } & I & Beta & $\mathbf{5}$ & $\mathbf{9}$ & $\mathbf{1 3}$ \\
Gamma & $0,054061^{*}$ & $0,016879^{\mathrm{ns}}$ & $0,025295^{\mathrm{ns}}$ & $0,048049^{\mathrm{ns}}$ \\
& Log Normal & $0,067458^{*}$ & $0,046430^{*}$ & $0,044371^{\mathrm{ns}}$ & $0,130881^{*}$ \\
& Normal & $0,070413^{*}$ & $0,057617^{*}$ & $0,051073^{*}$ & $0,139770^{*}$ \\
& Sb de Johnson & $0,061123^{*}$ & $0,024017^{\mathrm{ns}}$ & $0,031601^{\mathrm{ns}}$ & $0,110759^{*}$ \\
& Weibull & $0,059480^{*}$ & $0,026715^{\mathrm{ns}}$ & $0,032404^{\mathrm{ns}}$ & $0,106893^{*}$ \\
& Beta & $0,043749^{*}$ & $0,014132^{\mathrm{ns}}$ & $0,031883^{\mathrm{ns}}$ & $0,080094^{*}$ \\
\hline II & $0,012779^{\mathrm{ns}}$ & $0,043069^{*}$ & $0,020486^{\mathrm{ns}}$ & $0,048049^{\mathrm{ns}}$ \\
& Gamma & $0,026582^{*}$ & $0,040413^{*}$ & $0,036692^{*}$ & $0,130881^{*}$ \\
& Log Normal & $0,031697^{*}$ & $0,044587^{*}$ & $0,043781^{*}$ & $0,139770^{*}$ \\
& Normal & $0,015902^{\mathrm{ns}}$ & $0,037292^{*}$ & $0,023037^{\mathrm{ns}}$ & $0,110759^{*}$ \\
& Sb de Johnson & $0,017937^{\mathrm{ns}}$ & $0,037748^{*}$ & $0,024183^{\mathrm{ns}}$ & $0,106893^{*}$ \\
& Weibull & $0,012936^{\mathrm{ns}}$ & $0,041029^{*}$ & $0,023261^{\mathrm{ns}}$ & $0,080094^{*}$ \\
\hline III & Beta & $0,015105^{\mathrm{ns}}$ & $0,023388^{\mathrm{ns}}$ & $0,041033^{*}$ & $0,042900^{\mathrm{ns}}$ \\
& Gamma & $0,020224^{*}$ & $0,023626^{\mathrm{ns}}$ & $0,038803^{*}$ & $0,046017^{\mathrm{ns}}$ \\
& Log Normal & $0,022557^{*}$ & $0,023789^{\mathrm{ns}}$ & $0,044510^{*}$ & $0,045980^{\mathrm{ns}}$ \\
& Normal & $0,014923^{\mathrm{ns}}$ & $0,021677^{\mathrm{ns}}$ & $0,035086^{\mathrm{ns}}$ & $0,043462^{\mathrm{ns}}$ \\
& Sb de Johnson & $0,015472^{\mathrm{ns}}$ & $0,023769^{\mathrm{ns}}$ & $0,029636^{\mathrm{ns}}$ & $0,046520^{\mathrm{ns}}$ \\
& Weibull & $0,012154^{\mathrm{ns}}$ & $0,023073^{\mathrm{ns}}$ & $0,044606^{*}$ & $0,037023^{\mathrm{ns}}$ \\
\hline
\end{tabular}

${ }^{\text {ns: }}$ não significativo; ${ }^{*}$ : significativo ao nível de $5 \%$ de probabilidade.

Koehler et al. (2010) analisaram o efeito do sítio no desenvolvimento inicial de povoamento de araucária, bem como realizaram a seleção de funções densidade de probabilidade para a distribuição de diâmetros e alturas, por meio do teste de Kolmogorov-Smirnov. Esses autores destacaram que o sítio gera efeito significativo na distribuição de alturas, sendo a FDP de Weibull a mais indicada na descrição do histograma de alturas, sendo que quanto maior o valor de $\beta$, melhor qualificado é o sítio.

Nas tabelas 4 e 5 estão presentes as estatísticas de ajuste, precisão e coeficientes estimados para os três modelos de melhor desempenho geral no teste de Kolmogorov-Smirnov. De uma forma geral, a função Beta apresentou os melhores resultados para o IA e Syx\% em comparação às demais funções testadas, corroborando com o teste de aderência. A maior parte dos ajustes da função Beta proporcionou erros abaixo de $10 \%$ na estimativa do número de árvores por classe, com exceção aos ajustes para o $13^{\circ}$ ano em todos os sítios (Tabela 4). 
Tabela 4. Estatísticas de ajuste e precisão para as três melhores FDPs testadas.

Table 4. Statistics values of fitting and precision for the three best tested PDF.

\begin{tabular}{lccccccc}
\hline \multirow{2}{*}{$\begin{array}{l}\text { Idade } \\
\text { anos) }\end{array}$} & \multirow{2}{*}{ Sítio } & \multicolumn{2}{c}{ Beta } & \multicolumn{2}{c}{ Sb de Johnson } & \multicolumn{2}{c}{ Normal } \\
\cline { 2 - 8 } & IA & $\mathbf{S}_{\mathbf{y x}} \%$ & IA & $\mathbf{S}_{\mathbf{y x}} \%$ & IA & $\mathbf{S}_{\mathbf{y x}} \%$ \\
\hline 5 & I & 0,9917 & 5,33 & 0,9889 & 6,15 & 0,9889 & 6,15 \\
& II & 0,9990 & 2,88 & 0,9987 & 3,16 & 0,9989 & 2,97 \\
& III & 0,9967 & 4,05 & 0,9966 & 4,09 & 0,9978 & 3,31 \\
\hline 8 & I & 0,9973 & 3,21 & 0,9956 & 4,11 & 0,9960 & 3,90 \\
& II & 0,9981 & 3,89 & 0,9976 & 4,37 & 0,9977 & 4,25 \\
& III & 0,9989 & 2,56 & 0,9989 & 2,58 & 0,9989 & 2,52 \\
\hline 9 & I & 0,9929 & 4,94 & 0,9910 & 5,56 & 0,9913 & 5,47 \\
& II & 0,9974 & 4,57 & 0,9973 & 4,64 & 0,9977 & 4,27 \\
& III & 0,9953 & 6,12 & 0,9912 & 8,35 & 0,9915 & 8,21 \\
\hline 13 & I & 0,9807 & 11,24 & 0,9463 & 18,76 & 0,9530 & 17,56 \\
& II & 0,9784 & 12,76 & 0,9612 & 17,11 & 0,9618 & 5,74 \\
& III & 0,9917 & 6,62 & 0,9761 & 11,23 & 0,9807 & 10,11 \\
\hline
\end{tabular}

Tabela 5. Coeficientes estimados para as três melhores FDPs testadas.

Table 5. Estimated coefficients for the three best tested PDF.

\begin{tabular}{|c|c|c|c|c|c|c|c|c|c|c|c|c|c|}
\hline \multirow{3}{*}{$\begin{array}{l}\text { FDPs e seus } \\
\text { coeficientes }\end{array}$} & & \multicolumn{12}{|c|}{ Sítio } \\
\hline & & \multicolumn{4}{|c|}{ I } & \multicolumn{4}{|c|}{ II } & \multicolumn{4}{|c|}{ III } \\
\hline & & 5 & 8 & 9 & 13 & 5 & 8 & 9 & 13 & 5 & 8 & 9 & 13 \\
\hline \multirow[t]{4}{*}{ Beta } & $\alpha$ & 16,03 & 6,09 & 14,56 & 4,01 & 17,58 & 9,84 & 19,21 & 3,62 & 35,79 & 31,68 & 3,98 & 5,18 \\
\hline & $\beta$ & 9,39 & 3,32 & 6,61 & 1,28 & 11,42 & 5,67 & 14,95 & 1,91 & 34,16 & 67,56 & 2,10 & 1,58 \\
\hline & $\mathrm{x}_{\min }$ & 0,00 & 0,00 & 0,00 & 0,00 & 0,00 & 0,00 & 0,00 & 7,00 & 0,00 & 0,12 & 3,72 & 0,00 \\
\hline & $\mathrm{X}_{\max }$ & 17,73 & 22,26 & 23,00 & 22,00 & 15,35 & 18,75 & 22,69 & 19,00 & 14,13 & 34,12 & 14,50 & 14,33 \\
\hline \multirow{4}{*}{$\begin{array}{l}\text { Sb de } \\
\text { Johnson }\end{array}$} & $\mathrm{x}_{\min }$ & 4,50 & 0,00 & 0,00 & 0,00 & 0,00 & 0,46 & 0,00 & 4,49 & 0,00 & 0,00 & 3,97 & 0,00 \\
\hline & $\mathrm{x}_{\max }$ & 17,95 & 29,08 & 31,81 & 34,20 & 18,66 & 23,43 & 25,55 & 25,47 & 14,46 & 29,11 & 17,75 & 22,36 \\
\hline & $\gamma$ & 0,00 & 0,00 & 0,00 & 0,00 & 0,00 & 0,00 & 0,00 & 0,00 & 0,00 & 2,18 & 0,00 & 0,00 \\
\hline & $\delta$ & 1,95 & 2,10 & 3,45 & 2,11 & 3,31 & 2,47 & 3,30 & 2,11 & 4,26 & 4,28 & 1,61 & 2,53 \\
\hline \multirow[t]{2}{*}{ Normal } & $\boldsymbol{\mu}$ & 11,23 & 14,54 & 15,91 & 17,12 & 9,33 & 11,94 & 12,77 & 14,99 & 7,23 & 10,95 & 10,86 & 11,18 \\
\hline & $\sigma$ & 1,69 & 3,39 & 2,29 & 3,99 & 1,40 & 2,28 & 1,92 & 2,43 & 0,84 & 1,59 & 2,06 & 2,18 \\
\hline
\end{tabular}

Há um efeito positivo do sítio e da idade nos coeficientes estimados da função densidade de probabilidade Normal aplicada na distribuição de alturas de bracatingais (Tabela 5). Os valores dos parâmetros $\mu$ e $\sigma$ estimados tendem a aumentar conforme o aumento da idade e a melhora do sítio. Esse resultado foi observado por Machado et al. (2001), entretanto seu efeito sobre a forma e a amplitude da distribuição da variável não foram contemplados no trabalho.

O número de observações no ajuste dos modelos afetou de forma diferenciada a precisão alcançada pelas funções probabilísticas. Para a composição do histograma de frequências e ajustes das FDPs na idade de 13 anos, apenas 18 parcelas foram utilizadas. Isso se deve ao número reduzido de bracatingais existentes com essa idade, restringindo a amostra em termos de indivíduos por classe de sítio.

Entretanto, o número de observações, analisado isoladamente, não determina a aderência de um modelo probabilístico ao histograma de dados observados. O número de coeficientes do modelo, suas características matemáticas, as formas e amplitudes que pode simular, o método de ajuste, bem como a natureza dos dados utilizados para a modelagem são fatores determinantes na escolha de um modelo probabilístico.

Na figura 1 estão ilustradas as distribuições de frequências de alturas observadas nas diferentes idades e classes de sítio, bem como as distribuições estimadas pela função Beta. A distribuição de alturas do povoamento de bracatinga modifica ao longo do tempo, porém sempre se apresenta unimodal, mudando de uma assimetria positiva para uma negativa. 

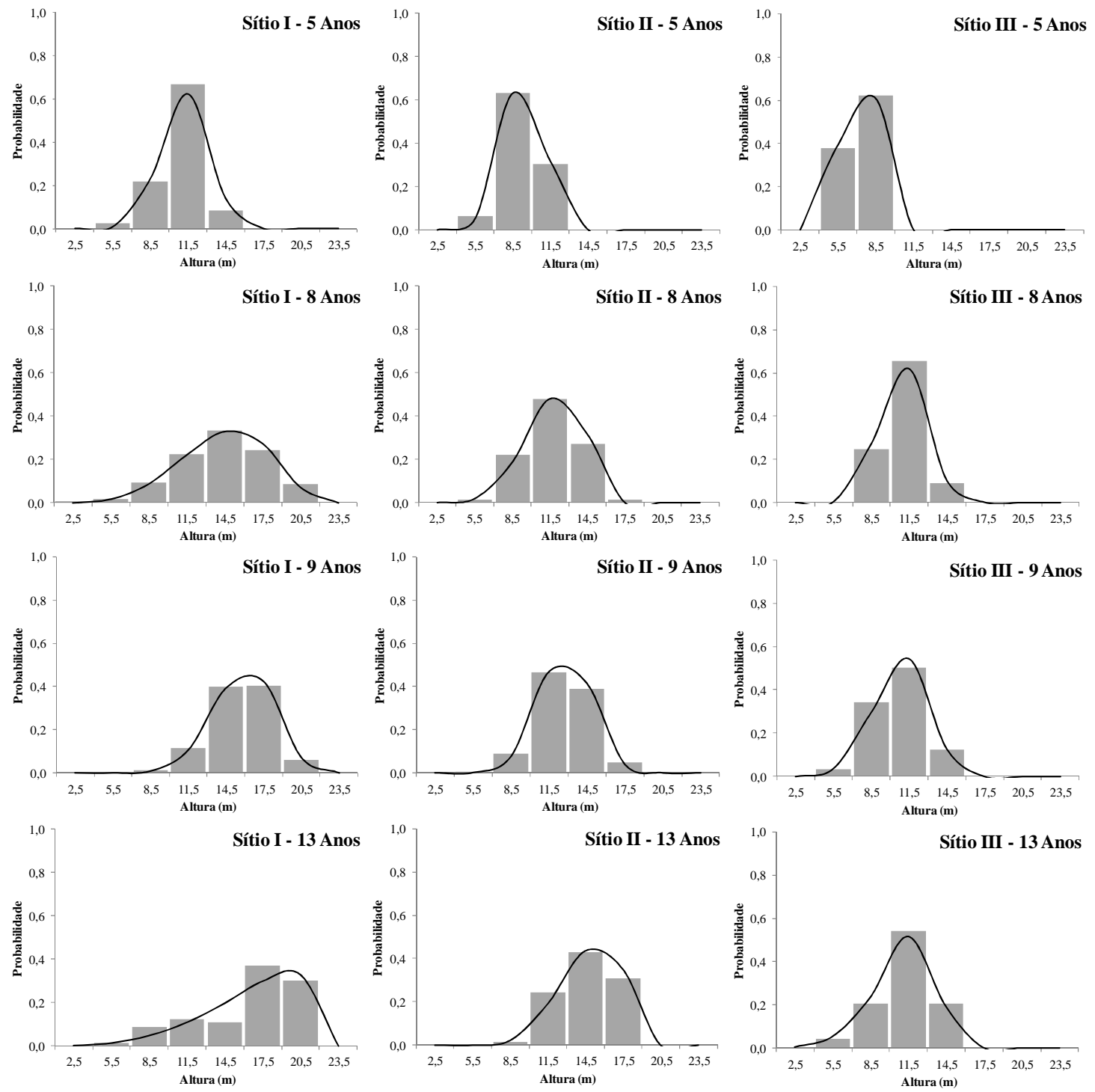

Figura 1. Frequência observada e estimada pela função Beta para a distribuição de altura de bracatingais. Figure 1. Observed and estimated frequencies by Beta function for the height distribution of bracatinga stands.

Essa dinâmica da distribuição se dá pelas características ecológicas da espécie e do sítio em que se encontra. Devido à bracatinga ser pioneira, dependente da incidência direta de luz sobre ela durante todo seu ciclo de vida, quando submetida a competição ao longo do processo de sucessão tem sua distribuição de indivíduos afetada conforme o sítio e o estágio de sucessão em que a floresta se encontra. Essa mudança na assimetria da distribuição é decorrente do efeito do sítio, em que, no sítio de melhor produtividade, a curva de distribuição das alturas se altera rapidamente quando comparado ao sítio de classe II, bem como o sítio de classe II se altera mais rápido quando comparado ao de classe III (Figura 1).

Espécies pioneiras são aqueles cujas sementes só germinam nas clareiras da floresta, a céu aberto e em plena luz solar, que incide ao nível do solo pelo menos durante parte do dia (SWAINE; WHITMORE, 1988). Devido à homogeneidade de alturas e à competição nos primeiros anos, há uma distribuição de alturas unimodal, próximo à normalidade, independentemente do sítio (Figura 1).

Entretanto, a competição gera a mortalidade dos indivíduos dominados e codominados ao longo do tempo, condicionando o povoamento a poucos indivíduos com acesso ao dossel da floresta, estes com altura 
média nas classes dominantes, tornando a distribuição de alturas assimétrica negativa no fim do ciclo de vida do povoamento, o que pode ser verificado ao se observar a idade de 13 anos (Figura 1). A velocidade dessas mudanças é dependente do sítio, e seu término se dá depois do início da segunda fase da sucessão florestal em ambientes naturais. Na figura 2 estão apresentados os histogramas modelados pela função densidade de probabilidade Beta apresentados por classe de sítio para todas as idades avaliadas.
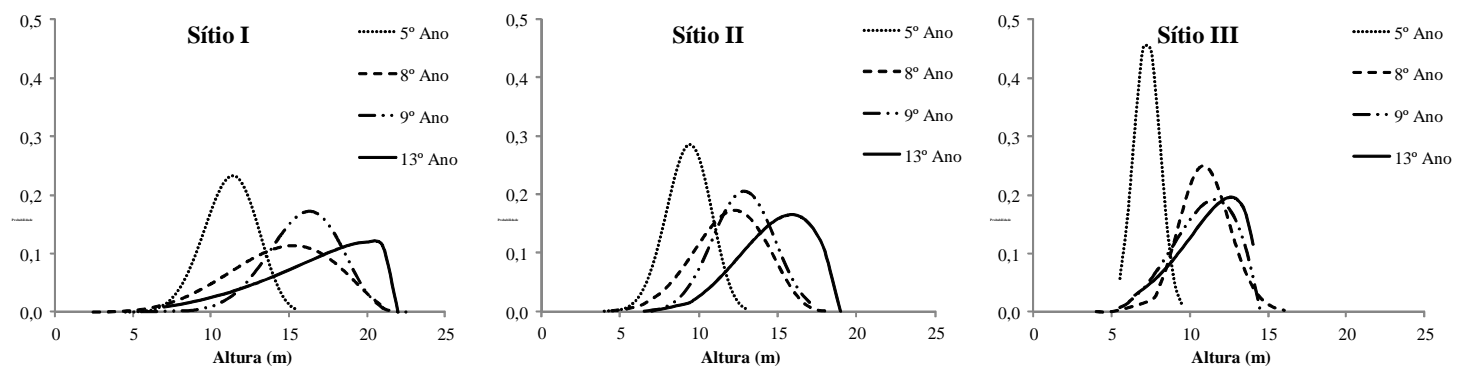

Figura 2. Distribuição de alturas obtidas a partir do ajuste da função Beta, nas diferentes idades e classes de sítio avaliadas.

Figure 2. Height distribution obtained from the adjustment of Beta function, at different ages and site evaluated classes.

O sítio afeta a distribuição das alturas dos povoamentos de bracatinga, seja essa mudança na forma ou na amplitude da distribuição, uma vez que nos sítios bons há maior competição, devido às melhores condições a que os indivíduos estão submetidos. Como consequência dessa competição, ocorre maior variância no porte dos indivíduos. Esse resultado pode ser facilmente observado na figura 2, na qual sítios bons apresentam distribuições platicúrticas, com maior desvio padrão, sendo portanto mais achatadas, e sítios ruins, distribuição de alturas leptocúrticas, em que os picos das curvas são mais elevados.

Machado et al. (2001) identificaram o efeito mais expressivo da idade no crescimento da variável altura nas idades iniciais. Os autores destacaram também que, nas idades entre 4 e 5 anos, a altura deixa de ser influenciada pela densidade. A idade, no entanto, afeta a altura mais expressivamente até aproximadamente 8 e 9 anos. O sítio, ao contrário dessas variáveis, exerce efeito significativo na distribuição das alturas até idades avançadas, sendo que, em povoamentos de sítios bons, a assimetria na distribuição é alcançada anteriormente aos demais sítios, devido às altas taxas de mortalidade nas classes inferiores.

\section{CONCLUSÕES}

- O sítio e a idade são variáveis que afetam a amplitude, forma e a dinâmica da distribuição das alturas de povoamentos de bracatinga da Região Metropolitana de Curitiba.

- A maior competição entre os indivíduos nos melhores sítios resulta em maior variância das alturas que, por sua vez, torna as curvas desses sítios platicúrticas, variando sua curtose para curvas leptocúrticas à medida que o sítio piora.

- A idade afeta a assimetria da distribuição das alturas. Em povoamentos novos, a assimetria é negativa ou não há assimetria, e em bracatingais velhos a assimetria é positiva, devido a um efeito direto dos aspectos ecológicos da espécie.

- A função densidade de probabilidade que melhor descreve a distribuição das alturas dos povoamentos de bracatinga em estudo é a Beta, podendo ser também representada pelas FDPs Sb de Johnson e a Normal em algumas combinações de idade e sítio. 


\section{REFERÊNCIAS}

ALVES JÚNIOR, F. T.; BRANDÃO, C. F. L. e S.; ROCHA, K. D. da; SILVA, J. T. da; MARANGON, L. C.; FERREIRA, R. L. C. Estrutura diamétrica e hipsométrica do componente arbóreo de um fragmento de Mata Atlântica, Recife-PE. Cerne, Lavras, v. 13, n. 1, p. 83 - 95, 2007.

BAGGIO, A. J.; CARPANEZZI, A. A.; GRAÇA, L. R.; CECCON, E. Sistema agroflorestal tradicional da bracatinga com culturas agrícolas anuais. Boletim de Pesquisa Florestal, Colombo, n. 12, p. 73 - 82, 1986.

BAGGIO, A. J. Estudio sobre el sistema agroforestal tradicional de la bracatinga (Mimosa scabrella Benth) en Brasil: productividad, manejo de residuos y elaboracion de compost. 1994. $242 \mathrm{f}$. Tesis (Doctoral) - Escuela Tecnica Superior de Ingenieros de Montes, Universidad Politecnica de Madrid, Madrid.

BARTOSZECK, A. C. P. S.; MACHADO, S. do A.; FIGUEIREDO FILHO, A.; OLIVEIRA, E. B. A distribuição diamétrica para bracatingais em diferentes idades, sítios e densidades na Região Metropolitana de Curitiba. Floresta, Curitiba, v. 34, n. 3, p. 305 - 323, 2004.

CARPANEZZI, A. A.; LAURENT, J. M. E.; CARVALHO, P. E. R.; PEGORARO, A.; BAGGIO, A. J.; ZANON, A.; OLIVEIRA, E. B. de; IEDE, E. T.; ROTTA, E.; STURION, J. A.; PEREIRA, J. C. D.; GRAÇA, L. R.; RAUEN, M. de J.; CARPANEZZI, O. T. B.; OLIVEIRA, Y. M. M. de. Manual técnico da bracatinga (Mimosa scabrella Benth.). Colombo: EMBRAPA-CNPF, 1988. 70 p. (Documentos, 20).

FELFILI, J. M. Diameter and height distributions in a gallery forest tree community and some of its main species in central Brazil over a six-year period (1985-1991). Revista Brasileira de Botanica, São Paulo, v. 20, n. 2, p. 155 - 162, 1997.

GOMIDE, L. R.; SCOLFORO, J. R. S.; OLIVEIRA, A. D. de. Análise das estruturas diamétricas e hipsométricas de fragmentos florestais localizados na bacia do rio São Francisco, em Minas Gerais. Floresta, Curitiba, v. 39, n. 2, p. 239 - 251, 2009.

GONÇALVES, M da P. M. Avaliação socioeconômica e ambiental de sistemas de produção de Mimosa scabrella Benth. e de Pinus taeda (L.). 153 f. Dissertação (Mestrado em Engenharia Florestal) Setor de Ciências Agrárias, Universidade Federal do Paraná, Curitiba, 2011.

KOEHLER, A. B.; CORAIOLA, M.; PÉLLICO NETTO, S. Crescimento, tendências de distribuição das variáveis biométricas e relação hipsométrica em plantios jovens de Araucaria angustifolia (Bertol.) Ktze., em Tijucas do Sul, PR. Scientia Forestalis, Piracicaba, v. 38, n. 85, p. 53 - 62, 2010.

MACHADO, S. A.; TONON, A. E. N.; OLIVEIRA, E. B.; FIGUEIREDO FILHO, A.; CARPANEZZI, A. A. Efeitos da densidade inicial e do sítio sobre o desenvolvimento de bracatingais nativos da Região Metropolitana de Curitiba. Boletim de Pesquisa Florestal. Colombo, n. 43, p. 19 - 46, 2001.

MACHADO, S. A.; NASCIMENTO, R. G. M.; MIGUEL, E. P.; TÉO, S. J.; AUGUSTYNCZIK, A. L D. Distribution of total height, transverse area and individual volume for Araucaria angustifolia (Bert.) $\mathrm{O}$. Kuntze. Cerne, Lavras, v. 16, n. 1, p. 12 - 21, 2010.

MACHADO, S, A.; SOUZA, R. F.; JASKIU, E.; CAVALHEIRO, R. Construction of site curves for native Mimosa scabrella stands in the Metropolitan Region of Curitiba. Cerne, Lavras, v. 17, n. 4, p. 489 - 497, 2011.

MICROSOFT CORPORATION. Microsoft Excel. Redmond, Wa, 2007.

PALUDO, G. F.; MANTOVANI, A.; KLAUBERG, C.; REIS, M. S. dos. Estrutura demográfica e padrão espacial de uma população natural de Araucaria angustifolia (Bertol.) Kuntze (Araucariaceae), na reserva genética florestal de Caçador, estado de Santa Catarina. Árvore, Viçosa, v. 33, n. 6, p. 1109 - 1121, 2009.

PUIG, H. A floresta tropical úmida. LOUREIRO, M. L. F. R. (Trad.). São Paulo: UNESP, 2008, 496 p. 
SCHLAEGEL, B. E. Testing, reporting and using biomass estimation models. In: Proceedings of the 1981 Southern Forest Biomass Workshop, 1., 1981, Clemson. Proceedings of... Clemson, 1981. p. 14.

SILVA, L. C. R. da. Estrutura e florística de bracatingais da Região Metropolitana de Curitiba, Paraná: um enfoque sucessional. 125 f. Dissertação (Mestrado em Ciências Florestais) - Universidade Federal do Paraná, Curitiba, 2013.

STEENBOCK, W. Domesticação de bracatingais: perspectivas de inclusão social e conservação ambiental. 262 f. Tese (Doutorado em Ciências, Área de Concentração Recursos Genéticos) - Centro de Ciências Agrárias, Universidade Federal de Santa Catarina, Florianópolis, 2009.

SWAINE, M. D.; WHITMORE, T. C. On the definition of ecological species groups in tropical rain forests. Vegetatio, v. 75, p. 81 - 86, 1988.

TÉO, S. J. Quantificação e modelagem do conteúdo de nutrientes na biomassa aérea de bracatinga (Mimosa scabrella Bentham). 156 f. Dissertação (Mestrado em Ciências Florestais) - Universidade Federal do Paraná, Curitiba, 2009.

WEBER, S. H. Desenvolvimento de nova função densidade de probabilidade para avaliação de regeneração natural. 2006. 74 p. Dissertação (Mestrado em Ciências Florestais) - Universidade Federal do Paraná, Curitiba, 2006. 
